\title{
Prenatal parental tobacco smoking, gene specific DNA methylation, and newborns size: the Generation R study
}

Marieke I. Bouwland-Both ${ }^{1,2}$, Nina H. van Mil ${ }^{1,2,3}$, Catharina P. Tolhoek ${ }^{1,2}$, Lisette Stolk ${ }^{4}$, Paul H. C. Eilers ${ }^{5}$, Michael M. P. J. Verbiest ${ }^{4}$, Bastiaan T. Heijmans ${ }^{6}$, André G. Uitterlinden ${ }^{4,7}$, Albert Hofman ${ }^{7}$, Marinus H. van ljzendoorn ${ }^{8}$, Liesbeth Duijts ${ }^{7,9}$, Johan C. de Jongste ${ }^{9}$, Henning Tiemeier ${ }^{3,7}$, Eric A. P. Steegers ${ }^{2}$, Vincent W. V. Jaddoe ${ }^{1,7}$ and Régine P. M. Steegers-Theunissen ${ }^{2^{*}}$

\begin{abstract}
Background: Deleterious effects of prenatal tobacco smoking on fetal growth and newborn weight are well-established. One of the proposed mechanisms underlying this relationship is alterations in epigenetic programming. We selected 506 newborns from a population-based prospective birth cohort in the Netherlands. Prenatal parental tobacco smoking was assessed using self-reporting questionnaires. Information on birth outcomes was obtained from medical records. The deoxyribonucleic acid (DNA) methylation of the growth genes IGF2DMR and H19 was measured in newborn umbilical cord white blood cells. Associations were assessed between parental tobacco smoking and DNA methylation using linear mixed models and adjusted for potential confounders.

Results: The DNA methylation levels of IGF2DMR and H19 in the non-smoking group were median (90\% range), $54.0 \%$ (44.6-62.0), and $30.0 \%$ (25.5-34.0), in the first trimester only smoking group $52.2 \%$ (44.5-61.1) and $30.8 \%$ (27.1-34.1), and in the continued smoking group $51.6 \%$ (43.9-61.3) and $30.2 \%$ (23.7-34.8), respectively. Continued prenatal maternal smoking was inversely associated with IGF2DMR methylation $(\beta=-1.03,95 \% \mathrm{Cl}-1.76 ;-0.30)$ in a dose-dependent manner $(P$-trend $=0.030)$. This association seemed to be slightly more profound among newborn girls $(\beta=-1.38,95 \%$ $\mathrm{Cl}-2.63 ;-0.14)$ than boys $(\beta=-0.72,95 \% \mathrm{Cl}-1.68 ; 0.24)$. $\mathrm{H} 19$ methylation was also inversely associated continued smoking $<5$ cigarettes/day $(\beta=-0.96,95 \% \mathrm{Cl}-1.78 ;-0.14)$. Moreover, the association between maternal smoking and newborns small for gestational age seems to be partially explained by IGF2DMR methylation ( $\beta=-0.095,95 \% \mathrm{Cl}-0.249 ;-0.018)$. Among non-smoking mothers, paternal tobacco smoking was not associated with IGF2DMR or H19 methylation.
\end{abstract}

Conclusions: Maternal smoking is inversely associated with IGF2DMR methylation in newborns, which can be one of the underlying mechanisms through which smoking affects fetal growth.

Keywords: Cigarettes, Cord blood, DNA methylation, Epigenetic epidemiology, H19, IGF2DMR, Maternal tobacco smoking, Paternal tobacco smoking

\footnotetext{
* Correspondence: r.steegers@erasmusmc.nl

2Department of Obstetrics and Gynecology, Erasmus MC, University Medical

Centre, Ee-building Room 2271a, PO Box 17383000DR, Rotterdam, The

Netherlands

Full list of author information is available at the end of the article
} source, provide a link to the Creative Commons license, and indicate if changes were made. The Creative Commons Public Domain Dedication waiver (http://creativecommons.org/publicdomain/zero/1.0/) applies to the data made available in this article, unless otherwise stated. 


\section{Background}

Impaired fetal growth and low birth weight increase the risk of short and long-term morbidity and mortality $[1,2]$. A suboptimal prenatal environment contributes to fetal endocrine and metabolic adaptations with permanent effects $[3,2]$. An important modifiable and adverse prenatal exposure is tobacco smoking by parents-to-be. The deleterious effects of maternal tobacco smoking on fetal growth and newborn weight are well-established [4]. The underlying mechanisms are not fully known but include vasoconstriction, reduced placental and fetal perfusion, and hypoxia [5]. Accumulating data suggest that derangements in epigenetic fetal and placental (re)programming may be one of the underlying mechanisms explaining the association between prenatal tobacco smoking and impaired fetal growth [6].

Epigenetic alterations are changes in gene expression potential that are not directly related to the deoxyribonucleic acid (DNA) sequence itself. DNA methylation modifications are one of the best understood epigenetic mechanisms [7]. During pregnancy, cells undergo major epigenetic reprogramming making them more susceptible for derangements during this period [7]. Both animal and human studies have shown that changes in the prenatal environment can lead to variations in epigenetic profiles [8-10]. Previous studies have shown global and gene specific differences in DNA methylation in different tissues in children prenatally exposed to tobacco smoking [11-13]. Insulin growth factor 2 (IGF2) and H19 are maternally and paternally imprinted genes located next to each other, playing an important role during fetal growth and development (Additional file 1: Figure S1) [14-17]. Moreover, the IGF2/H19 locus is a well-studied region that is known to be regulated through epigenetic mechanisms and sensitive to environmental exposures [9]. Previous research demonstrated that men may be more susceptible to detrimental early prenatal exposures than women [18].

Less is known about the influence of prenatal paternal tobacco smoking on gene-specific DNA methylation in newborns. Moreover, information on paternal tobacco smoking can be used to elucidate whether an effect on DNA methylation in newborns is due to the direct intrauterine effect of maternal smoking.

From this background, we aim to study whether prenatal parental tobacco smoking is associated with DNA methylation of IGF2 differentially methylated region $(D M R)$ and $H 19$ in umbilical white cord blood cells of newborns, with a focus on differences in sensitivity between genders. Furthermore, we examined mediation of DNA methylation in the association between tobacco smoking and the risk of being born small for gestational age (SGA).

\section{Results}

Maternal and newborns characteristics are presented in Table 1. Of the included mothers, $9.3 \%(n=47)$ reported tobacco smoking only in the first trimester and $24.7 \%(n=125)$ continued smoking during pregnancy. Mothers who continued tobacco smoking were younger, lower educated, and less often used a folic acid supplement during the periconception period than mothers who never smoked during pregnancy. Paternal tobacco smoking was more frequent in families with mothers who continued smoking $(72.8 \%$ in continued smoking versus $37.4 \%$ in non-smoking mothers). Median (90\% range) birth weights of newborns from non-smokers, first trimester only and continued smoking mothers were 3500 (2536-4383), 3410 (2154-4219), and 3195 (2348-4081) grams. The median ( $90 \%$ range) DNA methylation levels of IGF2DMR and $H 19$ were $53.2 \%$ (44.3-61.3) and $30.1 \%$ (25.6-34.1), respectively. IGF2DMR and H19 were correlated (Pearsons $r=0.147, P$ value $=0.001$ ). The DNA methylation levels of IGF2DMR and H19 in the non-smoking group were median (90\% range), $54.0 \%$ (44.6-62.0), and $30.0 \%(25.5-34.0)$, in the first trimester only smoking group $52.2 \%$ (44.5-61.1) and $30.8 \%$ (27.1-34.1), and in the continued smoking group $51.6 \%$ (43.9-61.3) and $30.2 \%$ (23.7-34.8), respectively. In addition, the methylation levels of IGF2DMR and $H 19$ were plotted according to each of the smoking categories (Additional file 2: Figure S2).

The associations between parental tobacco smoking and DNA methylation of IGF2DMR and $H 19$ are shown in Table 2. Although IGF2DMR methylation of mothers who smoked only during the first trimester showed a tendency to be lower compared to non-smokers, no significant differences were observed. However, continued prenatal smoking was associated with lower IGF2DMR methylation (crude model, $\beta=-1.14,95 \%$ CI $-1.81 ;-0.47, P$ value $=0.001$; adjusted model, $\beta=-1.03,95 \% \mathrm{CI}-1.76 ;-0.30, P$ value $=$ 0.006). Expressed as relative to the standard deviation, the difference found in the adjusted model corresponds with a standardized effect size in DNA methylation of -0.13 standard deviation score (SDS). Of the mothers who continued smoking, $77.6 \%(n=97)$ provided information concerning the amount of cigarettes they smoked, of which $42.3 \%(n=41)$ reported to have smoked less than five cigarettes per day and $57.7 \%(n=56)$ reported to have smoked five or more cigarettes per day. No significant association was found between the amount of cigarettes and IGF2DMR methylation. However, a dose-dependent association of the number of cigarettes smoked was assessed in mothers who continued smoking ( $P$ value for trend $=0.030$ ). Although no association was observed between mothers who continued smoking and H19 methylation, a significantly inverse association was revealed in mothers smoking up to five cigarettes per day (crude 
Table 1 Baseline characteristics

\begin{tabular}{|c|c|c|c|c|c|c|}
\hline \multirow[t]{3}{*}{ Characteristic } & \multicolumn{5}{|c|}{ Prenatal maternal tobacco smoking } & \multirow[b]{3}{*}{$P$ value } \\
\hline & \multirow[t]{2}{*}{ No $(n=334)$} & \multirow{2}{*}{$\begin{array}{l}\text { First trimester } \\
\text { only }(n=47)\end{array}$} & \multicolumn{3}{|l|}{ Continued } & \\
\hline & & & All $(n=125)$ & $\begin{array}{l}<5 \text { cigarettes per day } \\
(n=41)\end{array}$ & $\begin{array}{l}\geq 5 \text { cigarettes per day } \\
(n=56)\end{array}$ & \\
\hline \multicolumn{7}{|l|}{ Maternal } \\
\hline Age at intake (years), b & $31.5(22.5-38.1)$ & $30.5(20.1-38.3)$ & $28.5(19.0-37.7)$ & $29.4(20.0-37.5)$ & $28.4(18.4-39.9)$ & $<0.001$ \\
\hline $\begin{array}{l}\text { Body mass index at } \\
\text { intake }\left(\mathrm{kg} / \mathrm{m}^{2}\right)^{\mathrm{a}, \mathrm{b}}\end{array}$ & $23.1(19.5-32.2)$ & $23.2(19.6-33.1)$ & $23.7(18.2-32.5)$ & $22.8(18.2-33.6)$ & $24.8(18.0-33.9)$ & NS \\
\hline Education, $n(\%)$ & & & & & & $<0.001$ \\
\hline Primary education & $6(1.8)$ & $4(8.5)$ & $12(9.6)$ & $0(0)$ & $10(17.9)$ & \\
\hline Secondary education & $134(40.1)$ & $21(44.7)$ & $78(62.4)$ & $23(56.1)$ & $34(60.7)$ & \\
\hline Higher education & $193(57.8)$ & $22(46.8)$ & $33(26.4)$ & $17(41.5)$ & $11(19.6)$ & \\
\hline Missing & $1(0.3)$ & $0(0.0)$ & $2(1.6)$ & $1(2.4)$ & $1(1.8)$ & \\
\hline Parity (\%) & & & & & & NS \\
\hline 0 & $220(65.9)$ & $34(72.3)$ & $82(65.6)$ & $28(68.3)$ & $35(62.5)$ & \\
\hline$\geq 1$ & $114(34.1)$ & $13(27.7)$ & $43(34.4)$ & $13(31.7)$ & $21(37.5)$ & \\
\hline $\begin{array}{l}\text { Folic acid supplement use } \\
\text { during pregnancy, } n(\%)\end{array}$ & & & & & & $<0.001$ \\
\hline Start preconception & $188(56.3)$ & $18(38.3)$ & $29(23.2)$ & $11(26.8)$ & $15(26.8)$ & \\
\hline Start postconception & $84(25.1)$ & $19(40.4)$ & $36(28.8)$ & $13(31.7)$ & $13(23.2)$ & \\
\hline No & $25(7.5)$ & $7(14.9)$ & $33(26.4)$ & $10(24.4)$ & $14(25)$ & \\
\hline Missing & $37(11.1)$ & $3(6.4)$ & $27(21.6)$ & $7(17.1)$ & $14(25)$ & \\
\hline \multirow[t]{3}{*}{ Paternal smoking, $n(\%)$} & $125(37.4)$ & $28(59.6)$ & $91(72.8)$ & $14(34.1)$ & $9(16.1)$ & $<0.001$ \\
\hline & $<5$ cigarettes per day & $57(17.1)$ & $9(19.1)$ & $17(13.6)$ & $7(17.1)$ & $17(30.4)$ \\
\hline & $\geq 5$ cigarettes per day & $67(20.1)$ & $18(38.3)$ & $73(58.4)$ & $4(9.8)$ & $39(69.6)$ \\
\hline \multicolumn{7}{|l|}{ Newborns } \\
\hline Boys, $n(\%)$ & $195(58.4)$ & $21(44.7)$ & $81(64.8)$ & $26(63.4)$ & $34(60.7)$ & NS \\
\hline Birth weight ${ }^{a, b}$ & $3500(2536-4383)$ & 3410 (2154-4219) & 3195 (2348-4081) & $3175(2663-4252)$ & $3210(2334-4150)$ & $<0.001$ \\
\hline Gestational age at birtha, b & $40.3(37.6-42.0)$ & $40.3(36.5-41.9)$ & $40.1(37.1-42.5)$ & $40.6(37.0-42.9)$ & $40.2(36.9-42.3)$ & NS \\
\hline IGF2DMR methylation (\%) ${ }^{\mathrm{a}}$ & $54.0(44.6-62.0)$ & $52.2(44.5-61.1)$ & $51.6(43.9-61.3)$ & $52.0(44.0-61.2)$ & $51.1(41.2-63.9)$ & 0.033 \\
\hline H19 methylation (\%) ${ }^{1}$ & $30.0(25.5-34.0)$ & $30.8(27.1-34.1)$ & $30.2(23.7-34.8)$ & $29.8(21.5-33.0)$ & $30.2(23.1-37.0)$ & NS \\
\hline
\end{tabular}

Values are presented as median (90\% range) or as number (\%)

${ }^{\mathrm{b}}$ Missings; age at intake $(n=0)$, body mass index at intake $(n=1)$, gender $(n=0)$ birth weight $(n=0)$, gestational age at birth $(n=0)$

'ANOVA and chi-square tests are used to test differences between the different smoking categories

model, $-0.90,95 \%$ CI $-1.70 ;-0.11, P$ value $=0.026$; adjusted model, $\beta=-0.96,95 \% \mathrm{CI}-1.78 ;-0.14, P$ value $=$ 0.021). Expressed relative to the standard deviation, the difference found in the adjusted model corresponds with a standardized effect size in DNA methylation of -0.13 SDS. No significant associations were observed in mothers who reported to have smoked five or more cigarettes per day with H19 methylation. Among mothers who did not smoke during pregnancy, information on prenatal paternal tobacco smoking was provided by $99.4 \%$ of the mothers $(n=332 / 334)$. Of the fathers who smoked, $45.6 \%(n=57 / 125)$ smoked less than five cigarettes per day and $53.6 \%(n=67 / 125)$ smoked five or more cigarettes per day. No association was observed between paternal smoking and IGF2DMR or H19 methylation. We repeated the analyses after exclusion of the attention deficit hyperactivity disorder (ADHD) cases (see Additional file 3: Table S3), which did not change substantially the effect estimates.

Table 3 shows the associations between maternal tobacco smoking and DNA methylation, stratified by gender. In mothers who smoked during the first trimester, an inverse association among newborn girls was shown with IGF2DMR methylation (crude model, $\beta=-1.40$, $95 \%$ CI $-2.79 ;-0.01, P$ value $=0.048)$, which attenuated in the adjusted model $(\beta=-1.25,95 \% \mathrm{CI}-2.71 ; 0.21, P$ value $=$ $0.093)$. This association was not observed among boys. In addition, first trimester smoking was positively associated with $H 19$ methylation among girls but lost significance after adjustment (crude model, $\beta=1.04$, $95 \% \mathrm{CI}$ 
Table 2 Prenatal parental tobacco smoking habits and DNA methylation

\begin{tabular}{|c|c|c|c|c|c|c|}
\hline & \multicolumn{3}{|c|}{ IGF2DMR methylation } & \multicolumn{3}{|c|}{ H19 methylation } \\
\hline & bèta $^{a}$ & $95 \% \mathrm{Cl}$ & $P$ value & bèta $^{a}$ & $95 \% \mathrm{Cl}$ & $P$ value \\
\hline \multicolumn{7}{|c|}{ Model 1: adjusted for correlations between CpG sites, bisulphite batch, gestational age at birth } \\
\hline \multicolumn{7}{|l|}{ Maternal tobacco smoking } \\
\hline No $(n=334)$ & Referer & & & Refere & & \\
\hline First trimester only $(n=47)$ & -0.68 & $-1.67 ; 0.31$ & 0.178 & 0.61 & $-0.13 ; 1.35$ & 0.108 \\
\hline Continued smoking, all ( $n=125)$ & -1.14 & $-1.81 ;-0.47$ & 0.001 & -0.24 & $-0.75 ; 0.26$ & 0.348 \\
\hline$<5$ cigarettes per day $(n=41)$ & -1.06 & $-2.13 ;-0.00$ & 0.050 & -0.90 & $-1.70 ;-0.11$ & 0.026 \\
\hline$\geq 5$ cigarettes per day $(n=56)$ & -1.12 & $-2.06 ;-0.18$ & 0.019 & -0.13 & $-0.82 ; 0.56$ & 0.703 \\
\hline$P$ for trend & 0.006 & & & 0.298 & & \\
\hline \multicolumn{7}{|l|}{ Paternal tobacco smoking } \\
\hline No $(n=207)$ & Referer & & & Refere & & \\
\hline Yes $(n=125)$ & -0.24 & $-0.96 ; 0.48$ & 0.519 & -0.10 & $-0.61 ; 0.41$ & 0.707 \\
\hline$<5$ cigarettes per day $(n=57)$ & -0.26 & $-1.22 ; 0.70$ & 0.597 & -0.19 & $-0.87 ; 0.50$ & 0.592 \\
\hline$\geq 5$ cigarettes per day $(n=67)$ & -0.16 & $-1.06 ; 0.73$ & 0.719 & -0.04 & $-0.68 ; 0.60$ & 0.893 \\
\hline$P$ for trend & 0.643 & & & 0.795 & & \\
\hline
\end{tabular}

Model 2: model 1 + maternal characteristics (age, educational level, parity, BMI, periconception folic acid supplement use) and fetal gender Maternal tobacco smoking

\begin{tabular}{|c|c|c|c|c|c|c|}
\hline No $(n=334)$ & Referer & & & Referer & & \\
\hline First trimester only $(n=47)$ & -0.70 & $-1.71 ; 0.31$ & 0.175 & 0.56 & $-0.20 ; 1.32$ & 0.146 \\
\hline Continued smoking, all $(n=125)$ & -1.03 & $-1.76 ;-0.30$ & 0.006 & -0.37 & $-0.94 ; 0.20$ & 0.195 \\
\hline$<5$ cigarettes per day $(n=41)$ & -1.01 & $-1.89 ; 0.10$ & 0.069 & -0.96 & $-1.78 ;-0.14$ & 0.021 \\
\hline$\geq 5$ cigarettes per day $(n=56)$ & -0.89 & $-2.10 ; 0.08$ & 0.079 & -0.20 & $-0.93 ; 0.54$ & 0.602 \\
\hline$P$ for trend & 0.030 & & & 0.242 & & \\
\hline
\end{tabular}

Paternal tobacco smoking

\begin{tabular}{|c|c|c|c|c|c|c|}
\hline No $(n=207)$ & \multicolumn{3}{|c|}{ Reference } & \multicolumn{3}{|c|}{ Reference } \\
\hline Yes $(n=125)$ & -0.17 & $-0.91 ; 0.58$ & 0.663 & -0.23 & $-0.75 ; 0.30$ & 0.393 \\
\hline$<5$ cigarettes per day $(n=57)$ & -0.20 & $-1.18 ; 0.77$ & 0.681 & -0.23 & $-0.92 ; 0.45$ & 0.502 \\
\hline$\geq 5$ cigarettes per day $(n=67)$ & -0.07 & $-1.01 ; 0.88$ & 0.887 & -0.25 & $-0.91 ; 0.42$ & 0.46 \\
\hline$P$ for trend & \multicolumn{3}{|l|}{0.809} & \multicolumn{2}{|l|}{0.395} & \\
\hline
\end{tabular}

$0.04 ; 2.03, P$ value $=0.041 ;$ adjusted model, $\beta=1.00$, $95 \%$ CI $-0.05 ; 2.03, P$ value $=0.063)$. The association between continued maternal tobacco smoking and IGF2DMR methylation was slightly more profound among girls (crude model, $\beta=-1.46,95 \% \mathrm{CI}-2.59 ;-0.33, P$ value $=0.011$; adjusted model, $\beta=-1.38,95 \% \mathrm{CI}-2.63$; $-0.14, P$ value $=0.029$ ) than in boys (crude model, $\beta=-0.89$, $95 \%$ CI -1.73 ; $-0.06, P$ value $=0.037$; adjusted model, $\beta=-0.72,95 \% \mathrm{CI}-1.68 ; 0.24, P$ value $=0.142$ ).

Associations between maternal tobacco smoking, the risk of a newborn being SGA, and gene-specific DNA methylation were analyzed using mediation analyses. We hypothesized that epigenetic alterations can mediate the association between maternal prenatal tobacco smoking and impaired fetal growth (Fig. 1). The indirect effect, defined as the product of the coefficient for the effect of maternal tobacco smoking on DNA methylation (path a), and the coefficient for the effect of DNA methylation on the risk of a newborns being SGA (path b), was calculated (Fig. 2). The observed significant effect $(\beta=-0.095,95 \%$ CI -0.249 ; -0.018$)$ substantiates the notion that the association between maternal prenatal smoking and the risk of a newborn being SGA is partially mediated through $I G F 2 D M R$ methylation. The remaining direct effect of smoking on SGA (c') seems to be explained by the slightly younger age and lower educational level of the smoking 
Table 3 Prenatal maternal tobacco smoking and DNA methylation, stratified by gender

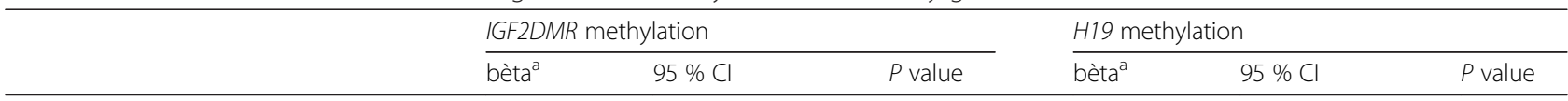

Model 1: adjusted for correlations between CpG sites, bisulphite batch, gestational age at birth

Boys

No $(n=195)$

First trimester only $(n=21)$

Continued smoking, all $(n=81)$

$P$ for trend

Girls
Reference

$\begin{array}{lll}0.12 & -1.33 ; 1.57 & 0.870 \\ -0.89 & -1.73 ;-0.06 & 0.037\end{array}$

0.044

Reference

$\begin{array}{lll}-1.40 & -2.79 ;-0.01 & 0.048 \\ -1.46 & -2.59 ;-0.33 & 0.011 \\ 0.005 & & \end{array}$

$$
0.005
$$

Reference

$\begin{array}{lll}0.18 & -0.95 ; 1.31 & 0.754 \\ -0.41 & -1.07 ; 0.25 & 0.222\end{array}$

0.249

Reference

$\begin{array}{lll}1.04 & 0.04 ; 2.03 & 0.041 \\ -0.10 & -0.90 ; 0.71 & 0.816 \\ 0.817 & & \end{array}$

$P$ for trend

Model 2: model 1 + maternal characteristics (age, educational level, parity, BMI, periconception folic acid supplement use)

Boys

$$
\text { No }(n=195)
$$

First trimester only $(n=21)$

Continued smoking, all $(n=81)$

$P$ for trend

Girls

No $(n=139)$

First trimester only $(n=26)$

Continued smoking, all $(n=44)$

$P$ for trend
Reference

$\begin{array}{lll}0.15 & -1.32 ; 1.61 & 0.845 \\ -0.72 & -1.68 ; 0.24 & 0.142 \\ 0.166 & & \end{array}$

0.166

Reference

$\begin{array}{lll}-1.25 & -2.71 ; 0.21 & 0.093 \\ -1.38 & -2.63 ;-0.14 & 0.029\end{array}$

0.018
Reference

$\begin{array}{lll}0.14 & -1.00 ; 1.28 & 0.810 \\ -0.69 & 1.45 ; 0.08 & 0.078 \\ 0.098 & & \end{array}$

Reference

$\begin{array}{lll}1.00 & -0.05 ; 2.03 & 0.063 \\ -0.05 & -0.93 ; 0.83 & 0.910\end{array}$

0.813

Results from linear mixed model analyses with maternal and parental tobacco smoking as independent variable and DNA methylation as dependent variable, stratified by gender

${ }^{a}$ Analyses were performed with square root transformed methylation data and values are presented as regression coefficients (95 \% confidence interval)

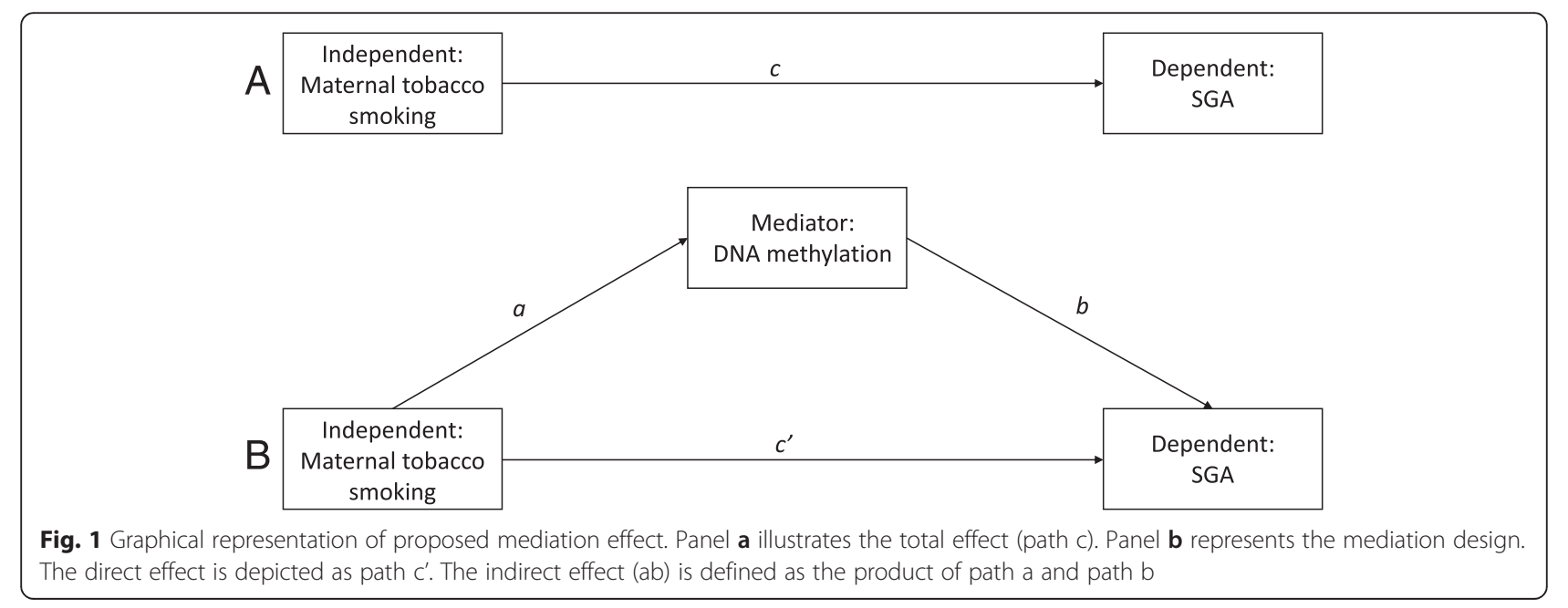



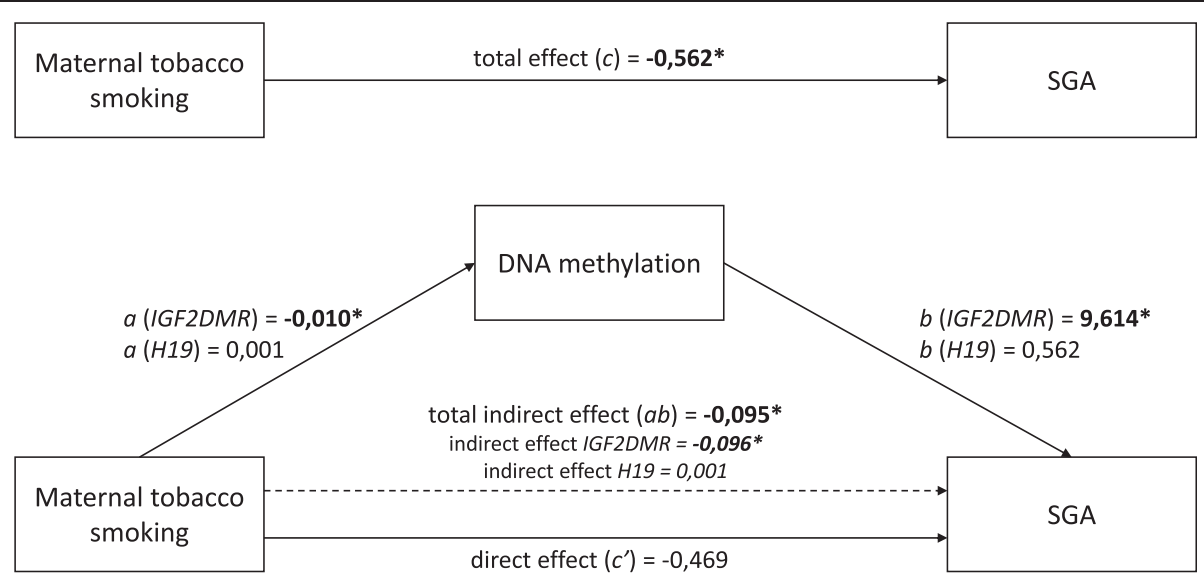

Fig. 2 Mediation analyses. Values represent unstandardized beta's. ${ }^{*} P<0.05$

mother. No use of a folic acid supplement, higher body mass index (BMI) of the mother, nulliparity, and the male gender of the newborn did not significantly explain this effect.

\section{Discussion \\ Main findings}

In 506 newborns, derived from a population-based birth cohort, we examined whether parental tobacco smoking is associated with IGF2DMR and H19 methylation in umbilical white cord blood cells. After adjustment for confounders, continued maternal prenatal tobacco smoking was associated in a dose-dependent manner with decreased IGF2DMR methylation in newborns. This association was stronger in newborn girls than boys. Decreased H19 methylation was also observed in mothers who continued to smoke $<5$ cigarettes/day. Prenatal paternal smoking was not associated with IGF2DMR or H19 methylation. Furthermore, the association between maternal smoking and the increased risk of being SGA may be partially mediated through IGF2DMR methylation.

\section{Interpretation}

Prenatal maternal tobacco smoking has been recognized as an important and modifiable detrimental exposure and has repeatedly been associated with disease $[4,19,5]$. Several studies have investigated associations between maternal prenatal smoking and global and gene-specific DNA methylation [20, 6]. Epigenome-wide methylation analyses revealed methylation changes at birth in newborns whose mother had smoked during pregnancy [21, 22]. Others investigated DNA methylation differences at the IGF2/H19 locus in relation to prenatal exposure to maternal tobacco smoking but found higher IGF2DMR methylation levels in boys born to smokers compared to non-smokers $(+4.7 \%$ IGF2DMR methylation) [15]. The region analyzed by Murphy et al. for the IGF2DMR included three CpG dinucleotides upstream of exon 3 (chr 11p15.5; CpG site 1, 2,169,518; CpG site 2, 2,169,515; and CpG site 3, 2,169,499; NCBI Human Genome Build 37/hg19) which overlapped with the region analyzed in our study (chr 11p15.5; CpG site 3, 2,169,709; CpG site 4, 2,169,660; CpG site 6/7, 2,169,518/2,169,515; NCBI Human Genome Build 37/hg19) [15]. The third CpG site analyzed by Murphy et al. overlapped with our eighth CpG site, which was excluded due to technical issues (see Additional file 4: Table S4) [15]. For the analyses of H19 DMR, the regions did not overlap. Other detrimental exposures, such as prenatal exposure to famine, have been previously linked to lower IGF2DMR methylation (-5.2 \% IGF2DMR methylation when periconception exposure to famine), whereas advantageous exposures, such as periconceptional folic acid supplement use, have been associated with increased methylation $(+4.5 \%$ IGF2DMR methylation) $[9,8]$. Our findings are also consistent with Guerrero-Preston et al., who also reported an inverse association between global DNA methylation and the nicotine biomarker cotinine however without stratification for gender [13]. In a previous study performed in the same population, we showed that SGA was associated with $-1.13 \%$ lower IGF2DMR methylation [17]. However, further research is warranted to further elucidate this difference.

In our study, the inverse association between maternal tobacco smoking from the first trimester onwards and IGF2DMR methylation seemed to be slightly more profound in girls. This finding is in contrast to previous research which demonstrated that boys may be more susceptible to detrimental exposures than girls [23, 18]. The first days after conception are important for the reprogramming of the epigenome. A limitation of our study is that we were not able to study difference in preconception and postconception smoke exposure on the methylation of the IGF2/H19 locus. This warrants further research. 
We have focused on the hypotheses that epigenetic alterations mediate the association between maternal tobacco smoking and adverse birth outcomes. Our study supports the notion that DNA methylation can be one of the mechanisms through which tobacco smoking impairs fetal growth. As nicotine crosses the placenta, it can directly harm the fetus [24]. Cigarette smoke has been shown to contain high levels of reactive oxygen species (ROS) causing oxidative stress [25]. DNA ROS damage can interfere with the binding of DNA methyltransferases (DNMTs) to the DNA, resulting in DNA hypomethylation [26]. Tobacco smoking can also indirectly damage the fetus through the effects on the placenta. Nicotine causes vasoconstriction and thus can impair placental function [27]. Increased vasoconstriction will limit blood flow and thus the provision of nutrients to the fetus, which could be compared to the effects of exposure to famine previously linked to epigenetic effects [9].

Mothers exposed to passive cigarette smoking show increased risks of adverse birth outcomes [28, 29]. Therefore, the strength of our study is that we were able to investigate direct intrauterine effects of maternal smoking by taking into account the effect of prenatal smoking of both parents. The effect of paternal smoking was not statistically significant and smaller than the effect of maternal smoking. This suggests that the observed effect is largely due to direct intrauterine effects of maternal smoking and not to unmeasured environmental factors as the mother and father are likely to share their environment, which is substantiated by a recent study [30].

DNA methylation is believed to be relatively stable throughout life, but these patterns are reprogrammed significantly during fetal and early postnatal life [7]. Therefore, it is assumed that the epigenome is susceptible for derangements during developmental stages when adverse prenatal environmental exposures can permanently alter methylation in the offspring. The periconception period is of particular importance, as epigenetic reprogramming takes most place during this period [7]. Our findings support the notion that changes in DNA methylation at the $I G F 2 D M R$ are being established during the periconception period, as the methylation levels of mothers who quit smoking during the first trimester showed a tendency to be lower than in non-smokers. The non-significance may also be explained by small numbers of mothers who quit smoking during the first trimester. However, a larger effect was observed in mothers who continued to smoke during pregnancy, indicating that these effects are not limited to the periconception period.

\section{Strengths and limitations}

This study was embedded in a large cohort from which a selection of Dutch newborn were studied. Selective participation did occur to some degree, as participating mothers were younger, more often single and smokers, and newborns were more often boys. This selection could have affected the external validity and generalizability of the study, especially when the associations would differ between the study population without and with the excluded newborns. This is difficult to ascertain, as the associations in the excluded group are unknown.

The study was based on a prospective data collection starting in early pregnancy. Detailed information was available on prenatal parental tobacco smoking habits, which enabled studying both trimester specific and dose-response associations. Although the use of questionnaires seems to be a valid method misclassification could have occurred, mostly leading to underreporting of tobacco smoking and therefore an underestimation of our estimates [31]. Lastly, although analyses were adjusted for potential confounders, the possibility of residual confounding cannot completely be excluded.

This study showed modest changes in DNA methylation in mothers who continued to smoke during pregnancy. Replication of these results is warranted. We were not able to assess whether the DNA methylation variations also resulted in changes in expression and long-term functional effects, although this has recently been shown in studies of others [32-34]. DNA methylation was measured in umbilical cord white blood cells and not in other tissues. It could be argued that DNA methylation patterns differ across various tissues [35, 36]. However, DNA methylation of imprinted genes such as $I G F 2 D M R / H 19$ is believed to be largely comparable in different tissues. For example, DNA methylation patterns of IGF2DMR in blood and in buccal cells and colon tissue showed moderate correlations $[35,37,36]$. We emphasize that it remains important to establish correlations between DNA methylation in peripheral tissues, e.g., blood, and tissues that are directly involved in the disease. However, in human epidemiological studies, it is commonly accepted to use blood or buccal samples due to relatively poor accessibility of other tissues.

\section{Conclusions}

Our study shows an inverse association of maternal prenatal tobacco smoking and its dose and duration with $I G F 2 D M R$ methylation in newborns. IGF2DMR methylation may mediate the association between maternal tobacco smoking and the risk of a newborn born SGA, supporting the notion that epigenetic alterations could be one of the mechanisms through which smoking affects fetal growth. The understanding how epigenetic control depends on early exposure may shed light on the link between fetal development and health over the life course. Our research emphasizes the importance to quit smoking preferably in the preconception period, although our data 
also shows that the advantageous effects of quitting smoking may not be limited to this period.

\section{Methods}

\section{Design and study population}

This study was embedded in the Generation R Study Rotterdam, the Netherlands, a population-based prospective cohort study from early pregnancy onwards [38]. The study has been approved by the Medical Ethical Committee of the Erasmus Medical Center in Rotterdam (MEC 198.782/2001/31). Written informed consent was obtained from all participating mothers for both maternal and child data. Mothers were enrolled during pregnancy, between 2001 and 2006. In total, 8880 mothers were enrolled during pregnancy.

For the present study, analyses were restricted to Dutch newborns, which were based on the country of birth of the parents and grandparents $(n=4882)$ [38]. In addition, newborns were selected with DNA extracted from umbilical cord white blood cells available $(n=3127)$. DNA methylation measurements were limited to 540 newborns due to logistic reasons. The current study is part of a project, in which we investigate the hypothesis that both SGA and children with ADHD have a shared causality in DNA methylation of especially imprinted fetal growth genes. The sampling strategy was to oversample the children born SGA or diagnosed with ADHD to improve the power of the analyses. Therefore, all infants born with a gestational age and sex-adjusted birth weight below -2 SDS $(n=69)$ were selected for analysis. Also, 92 children were included with ADHD based on parent interview Diagnostic Inventory of Screening Children or Child Behavior Checklist teacher report at age 6. Two of the infants with ADHD were also born with a gestational age and sex-adjusted birth weight below -2 SDS. The remaining 381 control newborns were randomly selected. Controls were born with a gestational age and sexadjusted birth weight above -2 SDS. Of the 540 newborns, data on parental smoking habits was available in $93.7 \%(n=506)$. The total population for analysis was 506 children (65 newborns born SGA, 88 cases of ADHD, and 441 control newborns). Characteristics of the included and excluded mothers and newborns were compared. Included versus excluded mothers were younger (30.3 years versus 31.1 years, $P$ value $<0.001$ ), smoked more often during pregnancy $(32.9 \%$ versus $26.0 \%, P$ value $<0.001)$, and were more often single $(10.9 \%$ versus $8.4 \%, P$ value $=0.02)$. Included versus excluded newborns were more often male $(58.7 \%$ versus $49.7(P$ value $<0.001)$ ). However, included and excluded participants were similar in terms of maternal BMI, maternal educational level, parity, folic acid supplement and alcohol use, and APGAR score after 1 and 5 min (all $P>0.05$ ).

\section{Prenatal parental tobacco smoking habits}

Information regarding maternal tobacco smoking was obtained by self-administered questionnaires sent in each trimester of pregnancy. Active maternal tobacco smoking at enrolment was assessed by asking the mother whether she smoked during pregnancy (no tobacco smoking, first trimester only tobacco smoking, continued tobacco smoking during pregnancy). This questionnaire was sent to all mothers, regardless of their gestational age at enrolment. In the second and third questionnaires, mothers were asked whether they smoked in the previous 2 months (no, yes). Mothers who reported that they did not smoke or only smoked during the first trimester, but still reported smoking in the second or third questionnaire, were classified as continuous smokers. Among the mothers who continuously smoked during pregnancy, the number of cigarettes were assessed and classified as (1) less than five cigarettes a day and (2) five or more cigarettes a day. Dose-response analyses for continuous smokers were based on the third trimester questionnaires. Prenatal paternal tobacco smoking was assessed at enrolment by asking the mother whether the father smoked during pregnancy (no, yes, do not know). Similar information was provided by the father in a subset of the participants $(n=433)$. Agreement between the two assessments was good (sensitivity $=92 \%$, specificity $=95 \%$ ). As more data was available from the mother questionnaire compared to the father questionnaire $(6.7 \%$ missing versus $19.8 \%$ missing, respectively), data obtained from the mother's questionnaire was used.

\section{Assessment of DNA methylation}

Genomic DNA was isolated from cord blood samples at birth as previously described [39]. We assessed DNA methylation of two imprinted loci, namely IGF2DMR and H19DMR. These loci were chosen based on their previously shown features of epigenetic regulation and their susceptibility for to environmental exposures [40]. Details of the measured amplicons can be found in Additional file 4: Table S4. Isolated genomic DNA (500 ng) was treated with sodium bisulphite for $16 \mathrm{~h}$ using the EZ-96 DNA methylation kit (Shallow) (Zymo Research, Irvine, CA, USA), according to the manufactures' protocol. Samples were randomly distributed on six 96well plates. The bisulphite treatment was followed by polymerase chain reaction (PCR) amplification, fragmentation after reverse transcription, and analysis on a mass spectrometer, according to the manufactures' protocol (MassARRAY EpiTYPER, Sequenom, Inc, San Diego, CA, USA). This generated mass signal patterns that were translated into quantitative DNA methylation levels of different CpG sites of the selected loci by MassARRAY EpiTYPER Analyzer software (v1.0, build1.0.6.88 Sequenom, Inc, San Diego, CA, USA) [41, 42]. Fragments containing 
one or more CpG sites were called CpG units. PCR and subsequent steps were performed in triplicate.

Data quality control for methylation consisted of exclusion of $\mathrm{CpG}$ units with too low or too high mass or CpG units with overlapping or duplicate RNA fragments (e.g., silent signals) were excluded from further analysis. Furthermore, at least two out of three of the replicate measurements per CpG unit had to be successful, the standard deviation of the duplicates or triplicates had to be $\leq 0.10$, and the success rate per CpG unit had to be $\geq 75 \%$. Also, from previous research it is known that PCR bias can occur with quantitative DNA methylation measurements methods [43]. To address this potential problem, we created standard curves constructed from DNA with low and high methylation (EpigenDx, Worcester, MA, USA) in steps of $10 \%$ methylation difference on both amplicons. CpGs showing irregularities were excluded from the analyses. Last, CpG units with interference of single nucleotide polymorphisms (CEU) with a frequency of $>5 \%$ were also excluded, as this could change the weight of the CpG unit and therefore interfere with the measurement. Details concerning the success rate of the amplicons can be found in Additional file 5: Table S5.

\section{Covariates}

From self-administered questionnaires, data was available on maternal age and maternal educational level, parity, and folic acid supplement use before and during pregnancy. Maternal education level was assessed by the highest completed education and classified as (1) none/primary or "low", (2) secondary or "medium", and (3) college/ university or "high". Parity was classified as (1) nulliparous and (2) multiparous. Folic acid supplement use was categorized into (1) folic acid supplement use (pre- or postconception start) and (2) no folic acid supplement use. At enrolment (median 13.5 weeks, 90 \% range 10.721.6), maternal weight and height were measured to calculate BMI $\left(\mathrm{kg} / \mathrm{m}^{2}\right)$. Information concerning date of birth, offspring sexm, and birth weight was obtained from community midwives and hospital registries.

\section{Statistical analyses}

Differences in maternal or newborns characteristics between maternal tobacco smoking categories were tested using ANOVA and chi-square tests. Thereafter, linear mixed models were used to examine the associations between maternal or paternal tobacco smoking (independent variable) and DNA methylation (dependent variable). This model was chosen as it can account for correlation between $\mathrm{CpG}$ dinucleotides, incorporates relevant adjustments within the models, and has the ability to accommodate missing data. The restricted maximum likelihood method was used for the model fitting. DNA methylation was treated as a continuous variable. To achieve normality, DNA methylation was square root transformed. Outliers per CpG (>3SDS) were excluded from further analysis. For all analyses, subject/person identifier was added as random effect and bisulphite batch and CpG site were added as fixed effects. In the crude analyses, maternal and paternal tobacco smoking were both entered as a fixed effects in separate models. In the adjusted analyses, potential confounders were additionally entered to the model at the same time as fixed effects.

Since maternal and paternal smoking are correlated, investigating paternal smoking among all mothers would overestimate the effect of paternal smoking if direct intrauterine mechanisms were present. Therefore, the association between paternal smoking and DNA methylation was examined among mothers who did not smoke during pregnancy. Prenatal exposures have shown to alter IGF2DMR methylation differently according to sex. [18] Therefore, the association between maternal tobacco smoking and DNA methylation was assessed in both boys and girls. The analyses were also repeated with exclusion of the ADHD $(n=88)$ cases. Two newborns were classified as both ADHD and SGA.

Additionally, we performed a mediation analyses to investigate whether DNA methylation of either IGF2DMR or $H 19$ mediates the association between maternal tobacco smoking and the risk of a newborns being born SGA. The direct and indirect effects between the dependent (SGA) and independent variable (maternal tobacco smoking) as well as the mediator (DNA methylation) were tested (Fig. 1) using a bootstrap approach (1000 samples) described by Preacher and Hayes [44].

Missing data of potential confounders (maternal educational level (0.6\%), maternal BMI (0.2\%), and maternal folic acid supplement use (13.2\%) were completed using the Markov-Chain-Monte-Carlo multiple imputation technique [45]. Ten imputed datasets were created. The linear mixed model analyses were performed using the data measured in triplicates including imputed missing data. Overall, the effect estimates using the imputed dataset were slightly smaller compared to the original dataset. All analyses were performed using the Statistical Package for the Social Sciences version 21.0 for Windows (SPSS Inc, Chicago, IL, USA).

\section{Additional files}

\footnotetext{
Additional file 1: Figure S1. Graphical representation of the IGF2DMR locus.

Additional file 2: Figure S2. Graphical representation of the Methylation levels of IGF2DMR and H19.

Additional file 3: Table S3. Parental tobacco smoking habits and DNA methylation, with exclusion of ADHD cases. Results from linear mixed model analyses with maternal or paternal tobacco smoking as independent variable and DNA methylation as dependent variable. ${ }^{1}$ Analyses were performed with square root transformed methylation
} 
data and values are presented as regression coefficients (95\% confidence interval). Analyses on paternal smoking were restricted to non-smoking mothers.

Additional file 4: Table S4. Details of measured amplicons and PCR primers. ${ }^{1}$ Genome built: GRch 37.67. ${ }^{2}$ Forward and reverse primer that will amplify the bisulphite converted genomic DNA. According to the MassARRAY EpiTYPER technology, taqs were added to the $5^{\prime}$ end of the primers. Forward primer: $10 \mathrm{mer}$ spacer tag is added at the $5^{\prime}$ primer end with the following sequence: 5'-AGGAAGAGAG + primer. Reverse primer: T7 promoter is added to the $5^{\prime}$ primer end with the following sequence: 5'-CAGTAATACGACTCACTATAGGGAGAAGGCT + primer.

Additional file 5: Table S5. Details quality control.

\section{Abbreviations}

ADHD: attention deficit hyperactivity disorder; BMI: body mass index; DMR: differentially methylated region; DNA: deoxyribonucleic acid; DNMT: DNA methyltransferases; IGF2: insulin growth factor-2; PCR: polymerase chain reaction; ROS: reactive oxygen species; SDS: standard deviation score; SGA: small for gestational age.

\section{Competing interests}

The authors declare that they have no competing interests.

\section{Authors' contributions}

MBB performed the experiments, carried out the statistical analyses, and was involved in the interpretation of the data. She wrote the first draft and revised all versions of the manuscript. NM performed the experiments and critically revised the manuscript. CT contributed to the statistical analyses and was involved in writing the first draft. LS and MV designed and supervised the experiments and critically revised the manuscript. PE contributed to the statistical analyses and interpretation of the data and critically revised the manuscript. $\mathrm{BH}$ advised in the epigenetic measurements and critically revised the manuscript. HT contributed to the design of the study and critically revised the manuscript. MI, LD, JJ, HT, AU, ES, AH, and VJ initiated and designed the original Generation R study and were responsible for the infrastructure in which the study is conducted. They contributed to the original data collection and critically revised the manuscript. RST initiated and designed the study. She supervised all aspects of the study and contributed to all revisions of the manuscript. All authors approved the final version of the manuscript.

\section{Acknowledgements}

The Generation R Study is conducted by the Erasmus Medical Center in close collaboration with the School of Law \& Faculty of Social Sciences of the Erasmus University Rotterdam; Municipal Health Service, Rotterdam; Rotterdam Homecare Foundation; and Stichting Trombosedienst \& Artsen laboratorium Rijnmond, Rotterdam. We gratefully acknowledge all participants and contributing general practitioners, hospitals, midwives, and pharmacies in Rotterdam.

\section{Funding}

The first phase of the Generation R study was made possible by financial support from the Erasmus Medical Center, Rotterdam, the Erasmus University Rotterdam, and the Netherlands Organization for Health Research and Development (ZonMw 21000074). MBB is financially supported by the Bo Hjelt Foundation (Grant 2009), which was not involved in any aspect of this study, i.e., design, statistical analyses, interpretation of the data, or writing and revising the manuscript.

\section{Author details}

'The Generation R Study Group, Erasmus MC, University Medical Centre Rotterdam, Rotterdam, The Netherlands. ${ }^{2}$ Department of Obstetrics and Gynecology, Erasmus MC, University Medical Centre, Ee-building Room 2271a, PO Box 17383000DR, Rotterdam, The Netherlands. ${ }^{3}$ Department of Child and Adolescent Psychiatry/Psychology, Erasmus MC, University Medical Centre, Rotterdam, The Netherlands. ${ }^{4}$ Department of Internal Medicine, Erasmus MC, University Medical Centre, Rotterdam, The Netherlands. ${ }^{5}$ Department of Biostatistics, Erasmus MC, University Medical Centre, Rotterdam, The Netherlands. ${ }^{6}$ Department of Molecular Epidemiology, Leiden University Medical Centre, Leiden, The Netherlands. ${ }^{7}$ Department of
Epidemiology, Erasmus MC, University Medical Centre, Rotterdam, The Netherlands. ${ }^{8}$ School for Pedagogical and Educational Sciences, Erasmus MC, University Medical Centre, Rotterdam, The Netherlands. ${ }^{9}$ Department of Pediatrics, Erasmus Medical Center, Rotterdam, The Netherlands.

Received: 25 April 2015 Accepted: 20 July 2015

Published online: 11 August 2015

\section{References}

1. Yanney M, Marlow N. Paediatric consequences of fetal growth restriction. Semin Fetal Neonatal Med. 2004;9(5):411-8.

2. Gluckman PD, Hanson MA, Cooper C, Thornburg KL. Effect of in utero and earlylife conditions on adult health and disease. N Engl J Med. 2008;359(1):61-73.

3. Steegers-Theunissen R, Twigt J, Pestinger V, Sinclair K. The periconceptional period, reproduction and long-term health of offspring: the importance of one-carbon metabolism. Hum Reprod Upd. 2013;19(6):640-55.

4. Jaddoe WW, Troe EJ, Hofman A, Mackenbach JP, Moll HA, Steegers EA, et al. Active and passive maternal smoking during pregnancy and the risks of low birthweight and preterm birth: the Generation R Study. Paediatr Perinat Epidemiol. 2008;22(2):162-71.

5. Bakker $\mathrm{H}$, Jaddoe WW. Cardiovascular and metabolic influences of fetal smoke exposure. Eur J Epidemiol. 2011;26:763-70.

6. Suter MA, Anders AM, Aagaard KM. Maternal smoking as a model for environmental epigenetic changes affecting birthweight and fetal programming. Mol Hum Reprod. 2013;19(1):1-6.

7. Nafee TM, Farrell WE, Carroll WD, Fryer AA, Ismail KM. Epigenetic control of fetal gene expression. BJOG. 2008;115(2):158-68.

8. Steegers-Theunissen RP, Obermann-Borst SA, Kremer D, Lindemans J, Siebel C, Steegers EA, et al. Periconceptional maternal folic acid use of 400 microg per day is related to increased methylation of the IGF2 gene in the very young child. PLoS One. 2009:4(11), e7845.

9. Heijmans BT, Tobi EW, Stein AD, Putter H, Blauw GJ, Susser ES, et al. Persistent epigenetic differences associated with prenatal exposure to famine in humans. Proc Natl Acad Sci U S A. 2008;105(44):17046-9.

10. Waterland RA, Jirtle RL. Transposable elements: targets for early nutritional effects on epigenetic gene regulation. Mol Cell Biol. 2003;23(15):5293-300.

11. Wilhelm-Benartzi CS, Houseman EA, Maccani MA, Poage GM, Koestler DC, Langevin SM, et al. In utero exposures, infant growth, and DNA methylation of repetitive elements and developmentally related genes in human placenta. Environ Health Perspect. 2012;120:296-302.

12. Breton $\mathrm{CV}$, Byun $\mathrm{H}$, Wenten $\mathrm{M}$, Pan F, Yang A, Gilliland FD. Prenatal tobacco smoke exposure affects global and gene-specific DNA methylation. Am J Respir Crit Care Med. 2009;180:462-7.

13. Guerrero-Preston R, Goldman LR, Brebi-Mieville P, Ili-Gangas C, Lebron C, Witter FR, et al. Global DNA hypomethylation is associated with in utero exposure to cotinine and perfluorinated alkyl compounds. Epigenetics. 2010;5:539-46.

14. Fowden AL, Sibley C, Reik W, Constancia M. Imprinted genes, placental development and fetal growth. Horm Res. 2006;65 Suppl 3:50-8.

15. Murphy SK, Adigun A, Huang Z, Overcash F, Wang F, Jirtle RL, et al. Genderspecific methylation differences in relation to prenatal exposure to cigarette smoke. Gene. 2012;494(1):36-43.

16. Soubry A, Murphy SK, Huang Z, Murtha AP, Schildkraut JM, Jirtle RL, et al. The effects of depression and use of antidepressive medicines during pregnancy on the methylation status of the IGF2 imprinted control regions in the offspring. Clin Epigenetics. 2011;3:2.

17. Bouwland-Both MI, van Mil NH, Stolk L, Eilers PH, Verbiest MM, Heijmans BT, et al. DNA methylation of IGF2DMR and H19 is associated with fetal and infant growth: the generation R study. PLoS One. 2013;8(12), e81731. doi:10.1371/journal.pone.0081731.

18. Tobi EW, Lumey LH, Talens RP, Kremer D, Putter H, Stein AD, et al. DNA methylation differences after exposure to prenatal famine are common and timing- and sex-specific. Hum Mol Genet. 2009;18(21):4046-53.

19. DiFranza JR, Aligne CA, Weitzman M. Prenatal and postnatal environmental tobacco smoke exposure and children's health. Pediatrics. 2004;113(4 Suppl):1007-15.

20. Obermann-Borst SA, Heijman BT, Eilers P, Tobi EW, Steegers EA, Slagboom PE, et al. Periconception maternal smoking and low education are associated with methylation of INSIGF in children at the age of 17 months. J Dev Orig Health Dis. 2012;3:315-20. 
21. Joubert BR, Håberg S, Nilsen R, Wang X, Vollset SE, Murphy SK, et al. 450K epigenome-wide scan identifies differential DNA methylation in newborns related to maternal smoking during pregnancy. Environ Health Perspect. 2012;120(10):1425-31.

22. Markunas CA, Xu Z, Harlid S, Wade PA, Lie RT, Taylor JA, et al. Identification of DNA methylation changes in newborns related to maternal smoking during pregnancy. Environ Health Perspect. 2014;122(10):1147-53. doi:10.1289/ehp.1307892

23. Pliushch G, Schneider E, Weise D, El Hajj N, Tresch A, Seidmann L, et al. Extreme methylation values of imprinted genes in human abortions and stillbirths. Am J Pathol. 2010;176(3):1084-90. doi:10.2353/ajpath.2010.090764.

24. Lambers DS, Clark KE. The maternal and fetal physiologic effects of nicotine. Semin Perinatol. 1996:20(2):115-26.

25. Faux SP, Tai T, Thorne D, Xu Y, Breheny D, Gaca M. The role of oxidative stress in the biological responses of lung epithelial cells to cigarette smoke. Biomarkers. 2009;14 Suppl 1:90-6.

26. Franco R, Schoneveld O, Georgakilas AG, Panayiotidis MI. Oxidative stress, DNA methylation and carcinogenesis. Cancer Lett. 2008;266(1):6-11.

27. Xiao D, Huang $X$, Yang S, Zhang $L$. Direct effects of nicotine on contractility of the uterine artery in pregnancy. J Pharmacol Exp Ther. 2007;322(1):180-5.

28. Salmasi G, Grady R, Jones J, McDonald SD. Environmental tobacco smoke exposure and perinatal outcomes: a systematic review and meta-analyses. Acta Obstet Gynecol Scand. 2010;89(4):423-41. doi:10.3109/00016340903505748.

29. Horta BL, Victora CG, Menezes AM, Halpern R, Barros FC. Low birthweight, preterm births and intrauterine growth retardation in relation to maternal smoking. Paediatr Perinat Epidemiol. 1997;11(2):140-51.

30. Joubert BR, Haberg SE, Bell DA, Nilsen RM, Vollset SE, Midttun O, et al. Maternal smoking and DNA methylation in newborns: in utero effect or epigenetic inheritance? Cancer Epidemiol Biomarkers Prev. 2014;23(6):1007-17. doi:10.1158/1055-9965.epi-13-1256.

31. Bakker $\mathrm{R}$, Kruithof $\mathrm{C}$, Steegers EA, Tiemeier H, Mackenbach JP, Hofman A, et al. Assessment of maternal smoking status during pregnancy and the associations with neonatal outcomes. Nicotine Tob Res. 2011;13(12):1250-6. doi:10.1093/ntr/ntr117.

32. Guida F, Sandanger TM, Castagne R, Campanella G, Polidoro S, Palli D, et al. Dynamics of smoking-induced genome-wide methylation changes with time since smoking cessation. Hum Mol Genet. 2015. doi:10.1093/hmg/ ddu751.

33. Richmond RC, Simpkin AJ, Woodward G, Gaunt TR, Lyttleton O, McArdle WL, et al. Prenatal exposure to maternal smoking and offspring DNA methylation across the lifecourse: findings from the Avon Longitudinal Study of Parents and Children (ALSPAC). Hum Mol Genet. 2014. doi:10.1093/hmg/ddu739.

34. Drake AJ, O'Shaughnessy PJ, Bhattacharya S, Monteiro A, Kerrigan D, Goetz S, et al. In utero exposure to cigarette chemicals induces sex-specific disruption of one-carbon metabolism and DNA methylation in the human fetal liver. BMC Med. 2015;13:18. doi:10.1186/s12916-014-0251-x.

35. Talens RP, Boomsma DI, Tobi EW, Kremer D, Jukema JW, Willemsen G, et al. Variation, patterns, and temporal stability of DNA methylation: considerations for epigenetic epidemiology. Faseb J. 2010;24(9):3135-44.

36. Herzog E, Galvez J, Roks A, Stolk L, Verbiest M, Eilers P, et al. Tissue-specific DNA methylation profiles in newborns. Clin Epigenetics. 2013;5(1):8.

37. Ally MS, Al-Ghnaniem R, Pufulete M. The relationship between gene-specific DNA methylation in leukocytes and normal colorectal mucosa in subjects with and without colorectal tumors. Cancer Epidemiol Biomarkers Prev. 2009;18(3):922-8.

38. Jaddoe WW, van Duijn CM, Franco OH, van der Heijden AJ, van lizendoorn MH, de Jongste JC, et al. The generation R study: design and cohort update 2012. Eur J Epidemiol. 2012;27(9):739-56.

39. Jaddoe WW, Bakker $R$, van Duijn CM, van der Heijden AJ, Lindemans J, Mackenbach JP, et al. The Generation R Study Biobank: a resource for epidemiological studies in children and their parents. Eur J Epidemiol. 2007;22(12):917-23.

40. Tobi EW, Slagboom PE, van Dongen J, Kremer D, Stein AD, Putter H, et al. Prenatal famine and genetic variation are independently and additively associated with DNA methylation at regulatory loci within IGF2/H19. PLoS One. 2012;7(5), e37933.

41. Coolen MW, Statham AL, Gardiner-Garden M, Clark SJ. Genomic profiling of CpG methylation and allelic specificity using quantitative high-throughput mass spectrometry: critical evaluation and improvements. Nucleic Acids Res. 2007;35(18), e119.
42. Ehrich M, Nelson MR, Stanssens P, Zabeau M, Liloglou T, Xinarianos G, et al Quantitative high-throughput analysis of DNA methylation patterns by base-specific cleavage and mass spectrometry. Proc Natl Acad Sci U S A. 2005;102(44):15785-90

43. Wojdacz TK, Borgbo T, Hansen LL. Primer design versus PCR bias in methylation independent PCR amplifications. Epigenetics. 2009;4(4):231-4.

44. Preacher KJ, Hayes AF. SPSS and SAS procedures for estimating indirect effects in simple mediation models. Behav Res Methods Instrum Comput. 2004;36(4):717-31.

45. Rubin DB, Schenker N. Multiple imputation in health-care databases: an overview and some applications. Stat Med. 1991;10(4):585-98.

\section{Submit your next manuscript to BioMed Central and take full advantage of:}

- Convenient online submission

- Thorough peer review

- No space constraints or color figure charges

- Immediate publication on acceptance

- Inclusion in PubMed, CAS, Scopus and Google Scholar

- Research which is freely available for redistribution 